\title{
Effects of low temperature heat release on the aerodynamics of a flat piston rapid compression machine: impact on velocity and temperature fields
}

\author{
Moez BEN HOUIDI*a, Julien SOTTON ${ }^{\mathrm{b}}$, Marc BELLENOUE ${ }^{\mathrm{b}}$, Camille STROZZI ${ }^{\mathrm{b}}$ \\ ${ }^{a}$ King Abdullah University of Science and Technology (KAUST), CCRC, PSE, Thuwal 23955-6900, Saudi Arabia. \\ ${ }^{b}$ ISAE-ENSMA, Institut Pprime, departement Fluide Thermique Combustion, BP 40109, Teleport2, 1 avenue Clement Ader, F86961 \\ Futuroscope Chasseneuil-du-Poitou Cedex, France.
}

\author{
*corresponding author. \\ E-mail adress: moez.benhouidi@kaust.edu.sa \\ Adress: Clean Combustion Research Center CCRC, building 5: Al Kindi West, R4216-WS17, King Abdullah \\ University of Science and Technology, Thuwal 23955-6900, Saudi Arabia.
}

Keywords: RCM, cool flame, two-stage auto-ignition, n-hexane, isooctane.

\begin{abstract}
The study of auto-ignition under temperature stratification is of great interest. Indeed, further understanding of the thermo-kinetic interactions and its influence on the combustion propagation regime is needed. In a previous work [1], experiments in a flat piston Rapid Compression Machine (RCM) demonstrated that the apparent propagation of reaction fronts is highly influenced by the typical temperature stratification observed at inert conditions. Nevertheless, the influence of low temperature heat release (LTHR) on the internal aerodynamics and temperature of the RCM is not well understood. In the present study, we first address the LTHR-flow interaction then address the LTHR-temperature interaction. We performed 2D-PIV experiments at $10 \mathrm{kHz}$ for inert and reactive lean isooctane mixtures. We averaged spatially the acceleration to present the time evolution during the cool flame period. We found that the normalized acceleration has a decreasing trend in both inert and reactive tests. No significant effect of the cool flame was observed on the trend. We performed temperature measurements using thin wire $(7.6 \mu \mathrm{m})$ type $\mathrm{K}$ thermocouples at inert and reactive n-hexane mixtures (same test conditions of fig.7 in [1]). The temperature evolution of the hot (adiabatically compressed) and the colder gases were recorded when cool flame occurs. The corrected gas temperature showed good agreement with the theoretical adiabatic core temperature as well as previous measurements with toluene LIF. In the tested case, we found that the cool flame induces an equal temperature rise of approximately $110 \mathrm{~K}$ in both the adiabatically compressed and the colder vortex gases. These results confirm quantitatively that LTHR does not significantly affect the mixing of the
\end{abstract}


temperature stratification of our flat piston RCM. In the studied test conditions, the temperature stratification is conserved globally despite the LTHR.

\section{Introduction}

Rapid Compression Machines (RCMs) have widely contributed to the understanding of fuels chemical kinetics. To this end, creviced pistons are often used to suppress the temperature heterogeneities generated by the roll-up vortices [2] [3]. The study of auto-ignition under temperature stratification is important for two key reasons: (i) understanding thermo-kinetic interaction is extremely helpful for the proper measuring of ignition delays with RCMs; (ii) understanding the influence of temperature stratification on the combustion regime and the pressure rise rate to improve the control of homogeneous charge compression ignition concepts.

In a previous study [1], we used the flat piston RCM of Pprime Institute (Poitiers) to address the abovementioned issues. Particularly, the temperature fields measured at inert condition were qualitatively used to interpret the combustion propagation regime of reactive mixtures. With single stage auto-ignition, it is commonly assumed that the temperature stratification is similar in inert and reactive tests [4]. However, with two-stage autoignition, the Low Temperature Heat Release (LTHR) may significantly affect the internal aerodynamics and local temperature of the RCM. In [1], we qualitatively demonstrated that LTHR does not significantly affect the aerodynamics of the roll-up vortices. We found it interesting to address this issue with a quantitative approach and to extend the analysis to the effects on temperature stratification.

Aerodynamics and temperature inside flat piston RCMs have been extensively studied with modeling [3] [5] and by experimentation [6] [7] [8] [9] [10] [11] [12] [13]. A few papers have experimentally studied the autoignition/turbulence interactions in RCMs. Guibert et al. [14] tested different fuels in the "Pierre and Marie Curie University" (UPMC) RCM where turbulence level can be varied. The authors demonstrated that the residual turbulence level significantly influences the combustion propagation regimes. Strozzi et al. [15] focused the analysis on the effects of the temperature gradients generated by the internal aerodynamic of the Pprime-RCM. The authors also found that the aerodynamics influence the onset of reaction fronts and deflagration regimes. Recently, Strozzi et al. [16] studied lean isooctane auto-ignition with high-speed PIV to analyze the combustion propagation regimes. The authors demonstrated that the reaction fronts propagation highly affected the velocity fields. To our knowledge, no studies have focused on the effect of the cool flame on aerodynamics.

The temperature inside the RCM has been extensively studied as it has a major impact on the auto-ignition chemistry. Yet, few experimental papers have focused on the influence of cool flame on the local temperature stratification in flat piston RCMs. In [17] [18] [19], Griffiths et al. used Rayleigh scattering from a 1D line to study 
temperature stratification during the auto-ignition of n-pentane and di-t-butyl peroxide in a flat piston RCM at various test conditions. Their analysis focused on the temperature stratification during the High Temperature Heat Release (HTHR). They demonstrated that the temperature stratification might be enhanced or destroyed according to the complex chemical kinetics. In single stage auto-ignition, temperature differences are rather enhanced. However, in two-stage auto-ignition, temperature difference may be smoothed if the reaction initiates first in the colder region due to negative temperature coefficient behavior.

In [6] [20], Desgroux et al. used Rayleigh scattering and thin wire thermocouples to study the temperature stratification in a flat piston RCM. Their measurements covered inert and reactive isooctane mixtures at different test conditions. The analysis of the temperature measurements were focused on the cool flame period. The authors concluded that heterogeneities are reduced prior to the ignition (HTHR).

Recently, laser absorption spectroscopy was applied to measure the temperature of reacting mixtures in RCMs equipped with creviced pistons [21] [22]. This technique can provide high time-resolved measurements without specific calibration [22]. Unfortunately, this technique provides measurements in the line-of-sight, which make it hard to use in devices with significant temperature heterogeneities.

The present study focuses on the influence of LTHR on both aerodynamics and thermal stratifications. First, we studied the effect of the cool flame on aerodynamics using a high-speed 2D-PIV technique under inert and reactive lean isooctane mixtures. Our results on the time evolution of the spatially averaged acceleration confirm the data presented in [1]. Secondly, we studied the temperature time evolution at two different locations in the RCM chamber. Measurements using thin-wire type $\mathrm{K}$ thermocouples at inert and reactive lean diluted n-hexane mixtures are corrected by considering the convective and radiative heat transfer errors. We interpreted the results of the reactive and inert tests based on the aerodynamic characterization of our RCM.

The assessment of LTHR-flow and LTHR-temperature are complementary. Indeed, our temperature measurement with thin-wire thermocouples at two locations was not spatially resolved. Thus, it was mandatory to address the effects of LTHR on the mixing of the observed temperature stratification. In both parts of this study, we performed the tests at comparable conditions. The PIV and temperature measurements were quite similar in terms of density and pressure. In both isooctane and n-hexane lean-reactive mixtures, we observed LTHR slightly after the End Of Compression (EOC).

To the best of our knowledge, this is the first study to focus on the cool flame behavior in a flat piston RCM using velocity and temperature measurements with a relatively high time resolution.

\section{Experimental set-up}


Experiments are performed in the flat piston RCM of Pprime Institute in Poitiers. The RCM has a squared section piston/cylinder of 50 x $50 \mathrm{~mm}$, a stroke of $420 \mathrm{~mm}$, a compression time of $35 \mathrm{~ms}$ approximately and can provide full optical access into the entire volume of the chamber at the EOC. The operation and typical postprocessing methods involved in this device are described in previous works [23] [24] [1] .

\subsection{Study of velocity fields under inert and reactive conditions}

In Table 1, we summarize the PIV test conditions. The reactive isooctane mixtures have a fuel/air equivalence ratio of 0.5 . The small variations of the initial temperature induced the observed differences in the adiabatic core temperature $T_{c}$. In Fig. 1, we present the averaged pressure profiles (from 5 tests), referencing the time scale to the EOC at the Top Dead Center (TDC). In all cases, the cool flame initiates slightly after TDC. The discrepancy of the ignition delays observed in Reactive $1 \mathrm{a}$ and $1 \mathrm{~b}$ cases is explained by the differences in $T_{c}$ and by the reproducibility of the mixture.

Table 1 : Summary of high-speed PIV test conditions. $\mathrm{P}_{\mathrm{c}}$ is the compression pressure.

\begin{tabular}{|c|c|c|c|c|c|c|}
\hline PIV tests & $\begin{array}{l}\text { Composition } \\
(\% \mathrm{~mol})\end{array}$ & Seeding & $\begin{array}{l}\mathbf{P}_{\mathbf{c}} \\
\text { (bar) }\end{array}$ & $\begin{array}{l}\text { Density } \\
\left(\mathrm{kg} / \mathrm{m}^{3}\right)\end{array}$ & $\begin{array}{l}\mathbf{T}_{\mathbf{c}} \\
(\mathbf{K})\end{array}$ & $\begin{array}{l}\text { Spatial } \\
\text { resolution } \\
(\mu \mathrm{m} / \mathbf{p x})\end{array}$ \\
\hline Inert 1 & $\begin{array}{l}\mathrm{N}_{2} / \mathrm{CO}_{2} / \mathrm{Ar} \\
58.2 / 23.5 / 18.3\end{array}$ & $\begin{array}{l}\text { Silicone oil droplets } \\
(3-9 \mu \mathrm{m})\end{array}$ & $\begin{array}{l}20.34 \pm \\
0.2 \%\end{array}$ & 12.2 & 732 & 48.8 \\
\hline Reactive 1a & $\begin{array}{l}\text { Isooctane } / \mathrm{N}_{2} / \mathrm{O}_{2} \\
0.8 / 78.4 / 20.8\end{array}$ & $\begin{array}{l}\text { Silicone oil droplets } \\
(3-9 \mu \mathrm{m})\end{array}$ & $\begin{array}{l}27.29 \pm \\
0.9 \%\end{array}$ & 14.4 & $712 \pm 3$ & 48.8 \\
\hline Reactive $1 \mathrm{~b}$ & $\begin{array}{l}\text { Isooctane } / \mathrm{N}_{2} / \mathrm{O}_{2} \\
0.8 / 78.4 / 20.8\end{array}$ & $\begin{array}{l}\mathrm{ZrO}_{2} \text { particles }(1-5 \\
\mu \mathrm{m})\end{array}$ & $\begin{array}{l}27.68 \pm \\
0.1 \%\end{array}$ & 13.4 & $727 \pm 3$ & 56.7 \\
\hline Reactive 2 & $\begin{array}{l}\text { Isooctane } / \mathrm{N}_{2} / \mathrm{O}_{2} \\
0.8 / 78.4 / 20.8\end{array}$ & $\begin{array}{l}\mathrm{ZrO}_{2} \text { particles }(1-5 \\
\mu \mathrm{m})\end{array}$ & $\begin{array}{l}20.01 \pm \\
0.3 \%\end{array}$ & 10.2 & $726 \pm 3$ & 56.7 \\
\hline
\end{tabular}

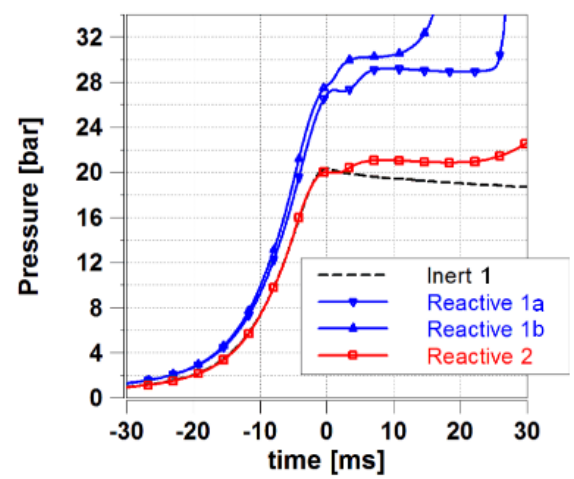

Figure 1 : Averaged pressure profiles (from 5 tests)

The PIV set-up for inert 1 and reactive 1a tests is detailed in [12], while the set-up for reactive $1 \mathrm{~b}$ and reactive 2 is detailed in [16]. We first used oil droplets seeding since this technique is easier to implement and allowed a better fidelity measurement. However, under reactive condition, oil droplets may evaporate and interact with autoignition chemistry. This motivated the use of zirconium-dioxide particles for the later reactive cases. Both seeding methods allowed a good measurement fidelity. The estimated Stokes number is approximately $6 \%$ for the heaviest 
particle and for the typical velocity level of $3 \mathrm{~m} / \mathrm{s}$ in the studied cases. In all tests, the same $10 \mathrm{kHz}$ PIV system is used and the laser sheet is placed in the RCM center plane. All tests are processed using the same cross-correlation method with an algorithm based on the time series pyramid correlation [25]. This was determined to be the optimal method to capture the wide range of velocities that we observed in the RCM. We used interrogation windows with decreasing size down to $32 \times 32$ and an overlap of $50 \%$. We post-processed the data to remove vectors with low peak ratio without applying any additional filters. We excluded missing vectors from the following result analysis.

\subsection{Local temperature measurements}

Thin wire thermocouples have been used to measure temperature under reacting conditions [20] [26] [27]. With careful design, the errors induced by conduction, convection and radiation heat losses may be reduced and thermocouples can have a high time response [28] [29] [30] [31] [32].

We used bare-bead and ground isolated type $\mathrm{K}$ thermocouples designed and mounted in Pprime Institute (Fig. 2). The sensor's wires have 2 diameters, $7.6 \mu \mathrm{m}$ and $200 \mu \mathrm{m}$. These wires fit the purity standards of type $\mathrm{K}$ thermocouples. Thus, we used a pre-calibrated acquisition system (QuickDAQ) to measure the temperature. According to the manufacturer, the accuracy of the measurement is within $\pm 1 \mathrm{~K}$. The hot junction, made on the 7.6 $\mu \mathrm{m}$ wires, has a rounded shape that does not excessively exceed the wire diameter (cf. Fig. 2). The distance separating the $200 \mu \mathrm{m}$ wires (L) is 400 times higher than the diameter of thin wires (d). The respect of the condition L > d make it reasonable to neglect the effects of conduction through the wires [33] [28]. This design provided high resistance to mechanical stress and a fast time response.

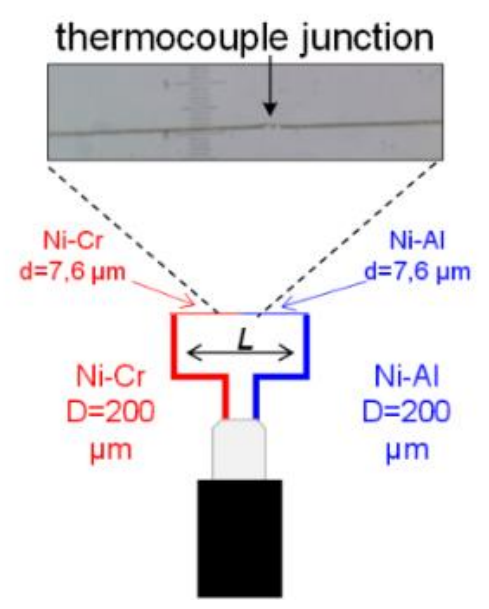

Figure 2 Schematic of the type $\mathrm{K}$ thermocouple design. A picture of the junction is presented above.

We corrected the measured junction temperature $\left(T_{j}\right)$ and calculated the gas temperature $\left(T_{g}\right)$ by [34]:

$$
\begin{aligned}
& T_{g}=T_{j}+\tau \frac{d T_{j}}{d t}+\frac{\sigma \varepsilon}{h}\left(T_{j}^{4}-T_{s}^{4}\right)(1) \\
& \tau=\frac{\rho_{j} c_{j} d_{j}}{4 h}
\end{aligned}
$$


$h=\frac{0.56 k U^{0.45}}{d_{j}^{0.55} v^{0.45}}$

In Eq. (1), $\sigma$ is the Stefan-Boltzmann constant, $\varepsilon$ is the temperature dependent emissivity $(\varepsilon=0.8-0.9$ according to [35]), $h$ is the convection heat-transfer coefficient, $T_{s}$ is the temperature of wall surfaces surrounding the junction (approximated by the measured wall temperature) and $\tau$ is the time constant of the convective heat-transfer contribution. In Eq. (2), $\rho_{j}$ is the thermocouple junction density, $c_{j}$ is the junction specific heat and $d_{j}$ is the junction diameter. For the convective heat-transfer coefficient, we used the approximation in Eq. (3) as described in [34]. In Eq. (3), $k$ is the gas thermal conductivity, $U$ is the gas velocity and $v$ is the kinematic viscosity.

We estimated the velocity based on PIV measurements performed in the RCM at the same compression ratio and at similar density levels. We used velocity time profiles measured at the location of the sensor in the chamber. With the careful design of the thermocouple and the relatively small junction size, the need for measurement correction is highly reduced (in order of few degrees in the post compression period, see Fig. 9). These sensors have an estimated correction lower than the uncertainty of the temperature measurement with toluene LIF technique (random error of $26 \mathrm{~K}$ is reported in [10] [1]).

Table 2 : Test matrix of the temperature measurements. $P_{c}$ and $T_{c}$ of reactive case are not reported since LTR initiate before the EOC, see [1].

\begin{tabular}{lllll} 
Tests & $\begin{array}{l}\text { Composition } \\
(\% \text { mol })\end{array}$ & $\begin{array}{l}\mathbf{P}_{\mathbf{c}} \\
(\mathbf{b a r})\end{array}$ & $\begin{array}{l}\text { Density } \\
(\mathbf{k g} / \mathbf{m 3})\end{array}$ & $\begin{array}{l}\mathbf{T}_{\mathbf{c}} \\
(\mathbf{K})\end{array}$ \\
\hline Inert 2 & $\mathrm{N}_{2} / \mathrm{CO}_{2} / \mathrm{Ar}$ & $11.12 \pm$ & 6.7 & 782 \\
& $21.9 / 15.6 / 62.5$ & $0.1 \%$ & & \\
\hline Reactive 3 & $\begin{array}{l}\text { n-hexane } / \mathrm{O}_{2} / \mathrm{N}_{2} / \mathrm{Ar} \\
0.7 / 16.1 / 27.8 / 55.4\end{array}$ & - & 6.5 & - \\
& 0.5 & & \\
\hline
\end{tabular}

In Table 2, we summarize the test conditions of the temperature measurements. We used the same test cases as in Sec. 4.1 in [1] where we discussed the impact of temperature stratification on the combustion propagation regimes. In Fig. 2, we present the pressure profile of the lean-diluted n-hexane mixture (fuel/air equivalence ratio of 0.4) and its equivalent inert mixture. The auto-ignition starts with a LTHR few milliseconds before the EOC. In [1], we used toluene LIF to measure temperature fields at the same inert test condition. At TDC, we demonstrated that the hot gases, which are adiabatically compressed, are located in the top and center of the chamber. However, the colder gases are located in the large-scale vortices at the bottom of the chamber. In the following, we refer to these regions as the "hot core" and the "colder vortex", respectively. Accordingly, we mounted the thermocouples in the RCM chamber to be able to measure the temperature of each region when LTHR occurs (see illustration in Fig. 3 and Fig. 4). 


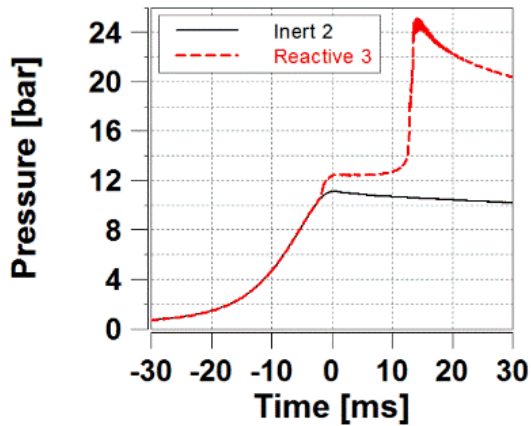

Figure 3 Pressure profiles of an inert and a reactive 3 tests.
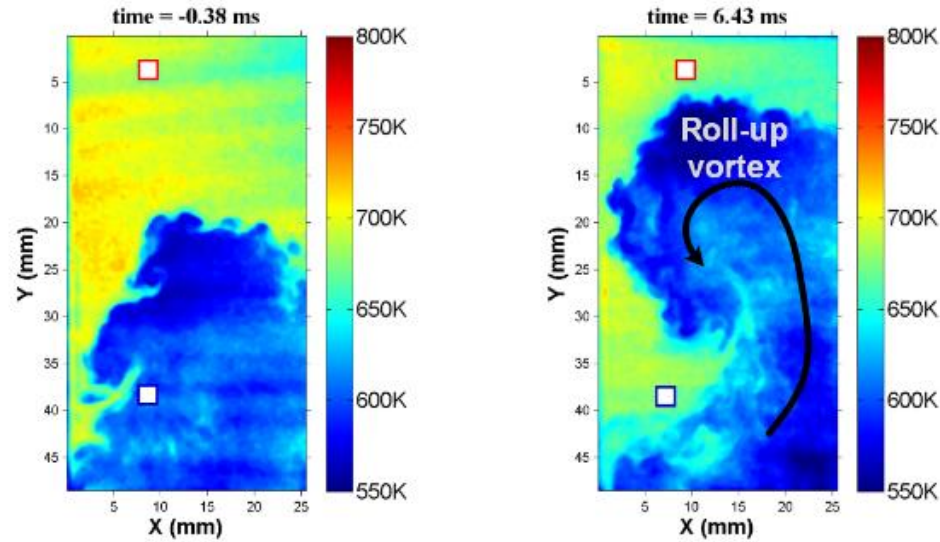

Location of thermocouples : $\square$ at the top, cylinder head side

$\square$ at the bottom, piston side

Figure 4 Illustration of the thermocouple positions in the reactive 3 case. The background images are temperature fields measured with toluene LIF at test conditions $\left(P_{c}\right.$ and $\left.T_{c}\right)$ similar to those of reactive 3 [1]. Time scale is referenced to TDC.

\section{Results}

\subsection{Effect of LTHR on aerodynamics}



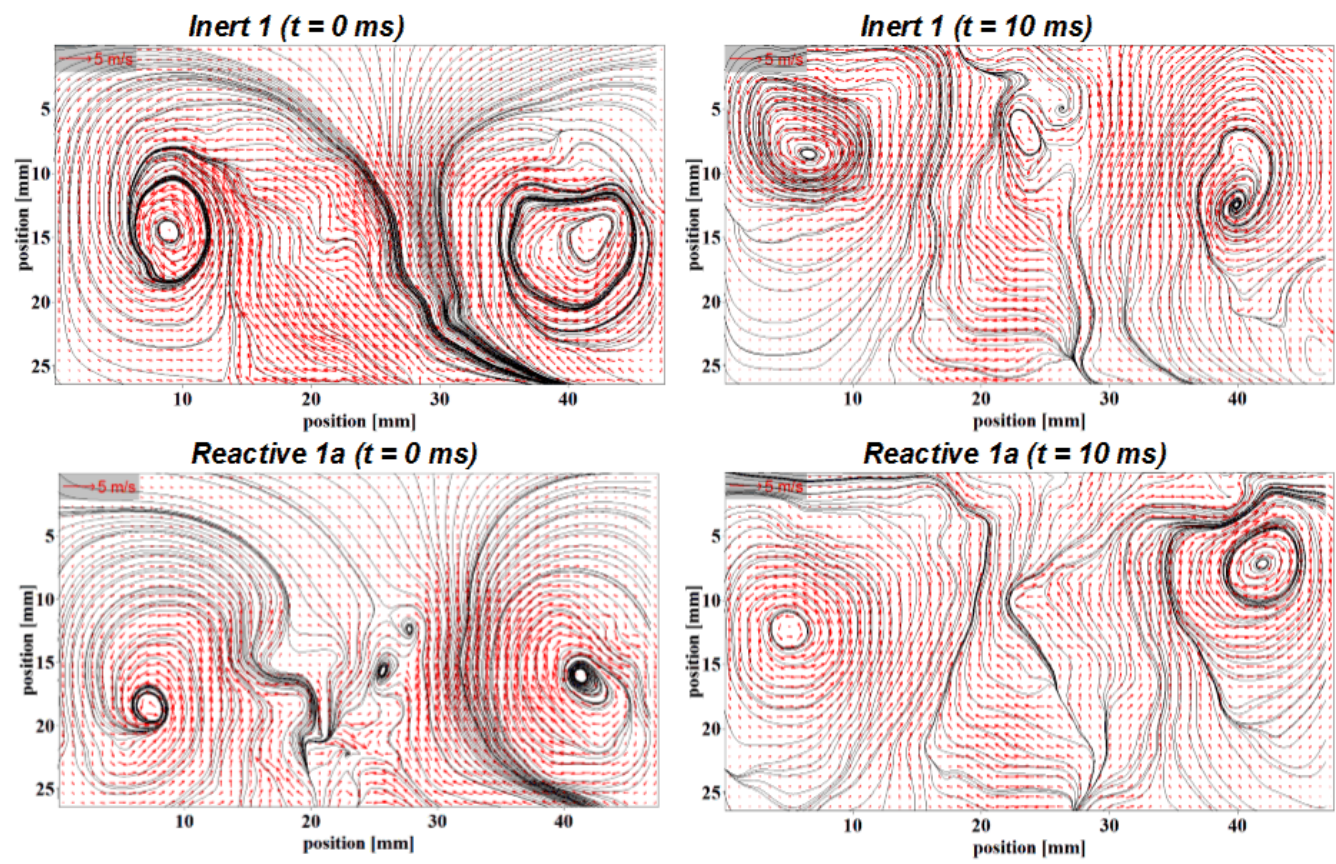

Figure 5 : Instantaneous velocity fields of an inert 1 and reactive 1 a tests.

In Fig. 5, we qualitatively compare the velocity fields of an inert 1 and a reactive 1 a tests, at TDC and $10 \mathrm{~ms}$ after TDC. Despite the slightly different compression pressure and density, the progression of the roll-up toroidal vortex toward the upper cylinder-head side of the chamber is similar in both cases. Due to test-to-test variation, the location of the large-scale vortices can be slightly different. We observed this similarly in both inert and reactive tests. The above qualitative PIV results confirm what we previously demonstrated with comparison of Schlieren images at the same test condition of Table 2 (cf. Fig. 8 in [1]). The roll up vortices continue to mix the reacting hot and colder vortex gases and seem unperturbed by the cool flame event.

To assess quantitatively the LTHR-flow interaction, we first studied the local velocity profiles and the turbulence. This approach was more interesting for the study of flow-combustion-regime interaction and should be discussed in a separate paper. In the present work, we found it more suitable to focus on the acceleration to illustrate specifically the flow-mixing interaction. We calculated the spatially averaged acceleration according to Eq. (4) and (5). We cycle averaged the resulting acceleration time profiles according to Eq. (6) ( $\mathrm{N}$ is the number of tests/cycles considered). Finally, we normalized the averaged acceleration relative to the value at TDC according to Eq. (7). This allowed an easier comparison between the inert and reactive cases since the average mass densities of these tests are slightly different.

$$
\begin{aligned}
& A=U^{\prime}(x, y, t, i) \\
& \bar{A}(t, i)=\frac{\sum_{x, y} A}{X . Y}
\end{aligned}
$$




$$
\begin{aligned}
& \overline{\bar{A}}(t)=\frac{\sum_{i} \bar{A}}{N} \\
& \hat{A}(t)=\frac{\overline{\bar{A}}}{\overline{\bar{A}}_{t=0}}
\end{aligned}
$$

In Fig. 6, Fig. 7 and Fig. 8, we present the results of the different reactive cases that we compare to the inert runs. Unless specified, all results are cycle averaged on five tests. We demonstrate that the normalized acceleration of the inert tests has a decreasing trend. At TDC, the RCM piston stops and the volume of the chamber is kept constant. It is likely that the viscous dissipation of the gas and in the walls boundaries that causes this behavior.

In Fig. 6 (respectively Fig. 8), we present the results of reactive 1a (respectively Reactive 2) tests. We plotted the averaged pressure trace to identify the LTHR and HTHR timings. However, in Fig. 7 we compare the cycle averaged inert 1 tests with a single reaction $1 \mathrm{~b}$ test. In both single and cycle averaged cases, we do not observe any significant change in the decreasing trend during the cool flame period. Conversely, HTHR induces a fast speed-up of the normalized acceleration reaching values one order of magnitude higher than those observed at TDC. In the post combustion period, we observe a faster decreasing rate. This is probably due to the increase in temperature and pressure. It seems that the low temperature reactions (LTRs) do not generate enough heat and/or the heat release rate is too low to cause perturbations on the global aerodynamics of our flat piston RCM.

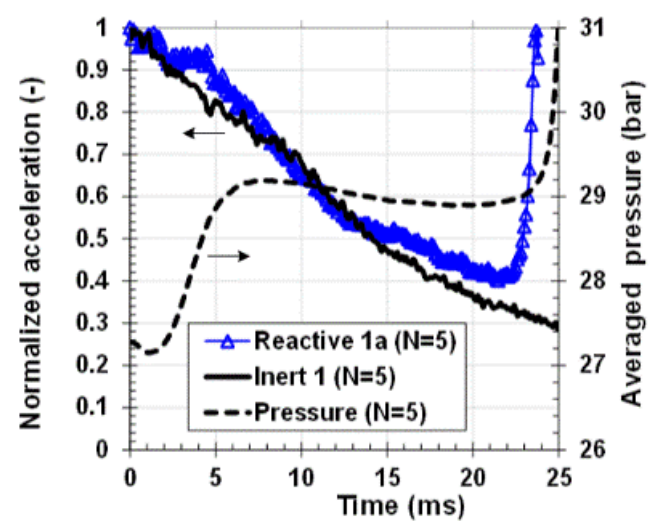

Figure 6 Time profiles of the spatially and cycle averaged acceleration. Dashed line represents the average pressure profile of the reactive tests. $\mathrm{N}$ is the number of tests considered. 


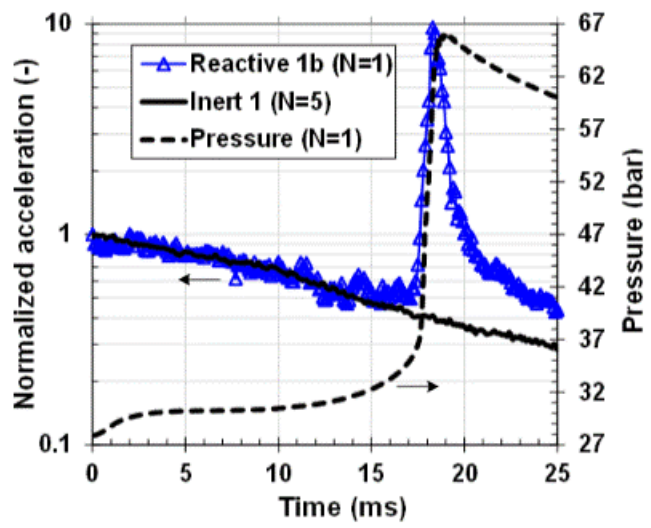

Figure 7 Time profiles of the spatially averaged acceleration. Dashed line represents the pressure profile of the reactive test. $\mathrm{N}$ is the number of tests considered.

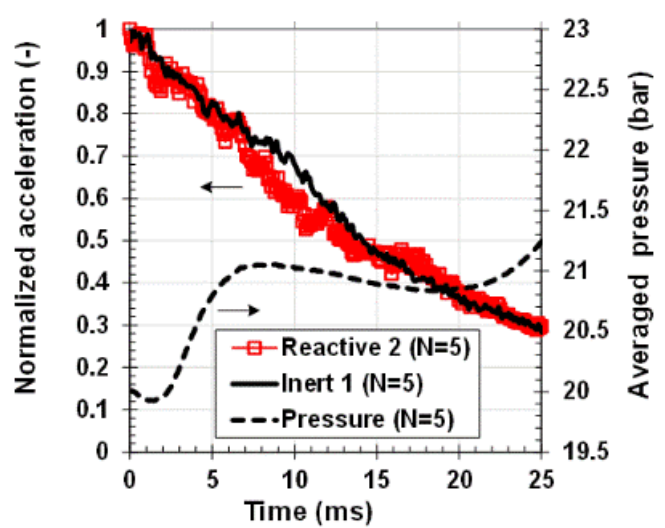

Figure 8 Time profiles of the spatially and cycle averaged acceleration. Dashed line represents the average pressure profile of the reactive tests. $\mathrm{N}$ is the number of tests considered.

\subsection{Effect of LTHR on temperature}

In Fig. 9, we plotted the measured $\mathrm{T}_{\mathrm{j}}$ and corrected temperature $\mathrm{T}_{\mathrm{g}}$ profiles of an example inert test. We compare the measurements with the calculated $T_{c}$ that represents the theoretical maximum temperature. In Fig. 10 (left), we compare the corrected temperatures of the inert 2 and the Reactive 3 tests. We present temperatures of the inert tests with averaged profile (thick lines, number of tests considered $N=9$ ) delimitated by the standard deviation $\pm \sigma$ (thinner lines) of test-to-test variations. We present the reactive tests (dashed lines) with averaged profile $(\mathrm{N}=2)$ delimitated by maximum and minimum values. The number of tests was limited as the temperature exceeded the calibration range of the sensor and caused the failure of the welding points. In Fig. 10 (right), we present the temperature difference between the two locations and we compare the results of inert and reactive tests. 


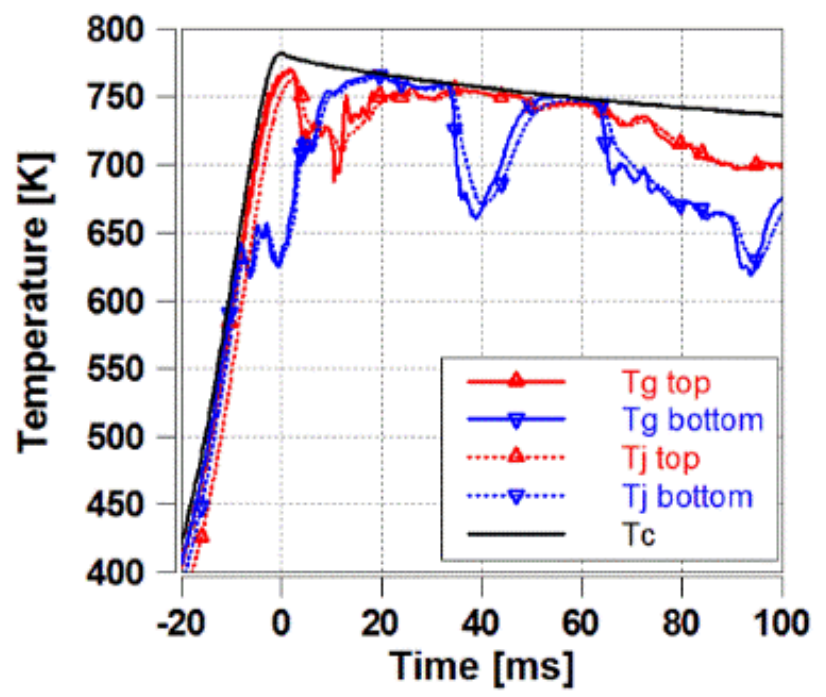

Figure 9 Comparison of measured and corrected temperature with theoretical adiabatic core temperature of one inert test.
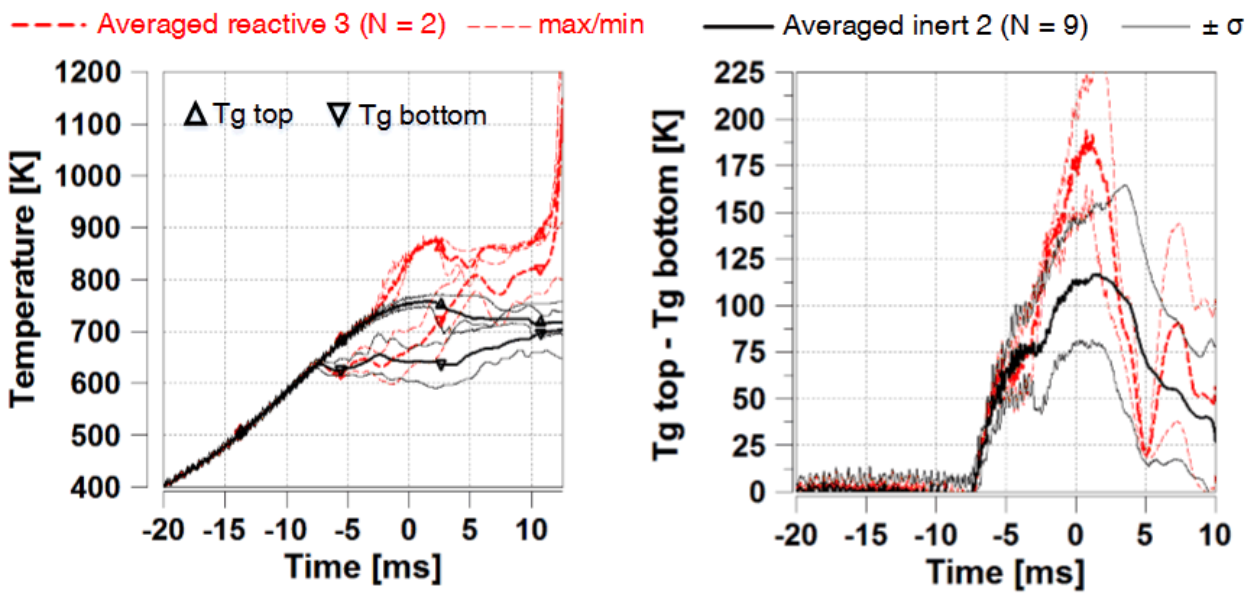

Figure 10 Comparison of corrected temperature measurements under inert 2 and reactive 3 conditions (left). Comparison of temperature difference, upper to lower location (right).

Results from inert 2 tests demonstrate that thermocouple measurements are in good agreement with $T_{c}$ calculations. The corrected temperature $T_{g}$ is always lower or equal to $T_{c}$. This also confirms that the adiabatic core hypothesis is valid up to approximately 60 to $70 \mathrm{~ms}$ after TDC for these test conditions. This is double the time than our previous estimation [1] based on quantitative toluene LIF. During the rapid compression, the fast increase in temperature is well predicted and thermocouples measure a temperature similar in both locations. This proves that these sensors have a relatively high time response. Approximately $7 \mathrm{~ms}$ before TDC, the temperature in the bottom of the chamber decreases. This corresponds to the instant at which the colder vortex gases reaches the location of the thermocouple in the bottom of the chamber. Up to $5 \mathrm{~ms}$ after TDC, the temperature is strictly higher 
in the top location. This demonstrates that around TDC, the thermocouples at the top and the bottom of the chamber are positioned in the adiabatically compressed and colder vortex regions, respectively. As illustrated in Fig. 4, the mixing induced by the internal aerodynamics of the RCM transports the core gases from the top and center of the chamber towards the bottom. This explains the observed variation of the temperature difference in Fig. 10, since the hot core gases cover both locations at 5 to $10 \mathrm{~ms}$ after TDC. Similar behavior was previously demonstrated in [10] where a higher compression ratio was used in the same RCM. The maximum temperature difference between the hot core and colder vortex gases is approximately $120 \mathrm{~K}$ (cf. Fig. 10). This is in excellent agreement with our previous measurements with toluene LIF [1] where we used the same test condition and the same RCM configuration. These results are also consistent with the values observed in flat piston RCMs reported in the literature [4] [9] [10].

In reactive 3 tests, the cool flame initiates when the top and bottom thermocouples measure, respectively, the temperature of the hot core and the colder vortex gases. The LTRs cause an equal increase of temperature in both locations. In Fig. 10, the temperature difference has a similar time profile compared to the inert behavior. Hence in the present test conditions, temperature increases similarly in both hot region and colder vortex regions. The temperature increase is approximately $110 \mathrm{~K}$, which is consistent with reported values of typical two-stage autoignition in RCMs; see for instance [22] where the measured increase during the first stage ignition of n-pentane was $110-130 \mathrm{~K}$ according to the test conditions. It is worth noting that the HTHR initiate when the temperature of the core gases reaches approximately $900 \mathrm{~K}$.

In [17] [18] [19], Griffiths et al. pointed out that the onset of LTRs may smooth the temperature stratification observed in flat piston RCMs. Obviously, this is not the case in two stage auto-ignition when both hot core and colder vortex regions feature LTRs. The present work demonstrate this. However, in some cases auto-ignition may first occur in colder gases due to negative temperature coefficient (NTC) behavior. As demonstrated in the literature [17] [36] [1], the auto-ignition in colder region can induce an earlier onset of the overall thermal runaway. The cool flame has always a positive temperature coefficient behavior. Consequently, LTRs are expected to initiate in regions of higher temperature. At temperatures near to the higher limit of the NTC range, the adiabatically compressed gases may auto-ignite with a single stage chemistry, whereas LTRs occur in the colder vortex region and cause a shorter ignition delay. This would lead to an increase in the temperature of the colder region as opposed to unperturbed temperature in the core gases. In such specific cases, the initial temperature stratification may be highly smoothed. If we assume that the combustion propagation regime is mainly with reaction fronts in such thermo-kinetic conditions, then the propagation speed would be proportional to the inverse 
of ignition delay gradients, as proposed by Zeldovich [37]. Thus, when the ignition delays of the hot core and colder vortex regions are relatively similar, the combustion propagation speed substantially increases. This may induce a high pressure rise rate and possibly a high knock levels.

\section{Conclusion}

We addressed the influence of cool flame on the aerodynamics of a flat piston RCM. First, we studied the internal aerodynamics under inert and reactive isooctane conditions. Using qualitative and quantitative analysis, we demonstrated that the LTHR did not significantly affect the aerodynamics to an extent detectable by our experimental set-up. Despite LTRs, the toroidal roll-up vortex keeps mixing the reacting hot and colder gases as in the inert test conditions.

Based on our understanding of the internal aerodynamics of the Pprime flat piston RCM, we conducted temperature measurements with thin-wire thermocouples at inert and reactive n-hexane test conditions. We chose test conditions in which two separate sensors were able to measure the temperature increase of the hot core and the colder vortex gases when LTHR occurs. The thermocouples had a fast time response and were able to measure the temperature fluctuation in the compression and post compression period with a good precision. We demonstrate that LTHR induces an equal increase of the temperature in hot and colder gases.

\section{Acknowledgement}

We thank Mr. Herve Arlaud and Dr. Gildas Lalizel for providing the thermocouples for this study. We thank Mr. Alain Claverie and Mr. Antoine Delicourt for their assistance in the PIV experiments. We acknowledge the financial support of ANRT (PN: ANR-PLASMAFLAME-2011BS0902501).

\section{References}

[1] M. Ben-Houidi, J. Sotton and M. Bellenoue, "Interpretation of auto-ignition delays from RCM in the presence of temperature heterogeneities: Impact on combustion regimes and negative temperature coefficient behavior," Fuel, vol. 186, pp. 476-495, 2016.

[2] C.J. Sung and H.J. Curran, "Using rapid compression machines for chemical kinetics studies," Progress in Energy and Combustion Science, vol. 44, pp. 1-18, 2014.

[3] S. S. Goldsborough, S. Hochgreb, G. Vanhove, H. J. C. M. S. Wooldridge and C. J. Sung, "Advances in rapid compression machine studies of low- and intermediate-temperature autoignition phenomena," Progress in Energy and Combustion Science, vol. 63, pp. 1-78, 2017.

[4] J. Clarkson, J.F. Griffiths, J.P. Macnamara and B. Whitaker, "Temperature fields during the development of combustion in a rapid compression machine," Combustion and Flame, vol. 125, no. 3, pp. 1162-1175, 2001.

[5] G. Mittal, M. P. Raju and C.J. Sung, "CFD modeling of two-stage ignition in a rapid compression machine: Assessment of zero-dimensional approach," Combustion and Flame, vol. 157, pp. 1316-1324, 2010.

[6] P. Desgroux, L. Gasnot and L. Sochet, "Instantaneous temperature measurement in a rapid-compression machine using laser rayleigh scattering," Applied Physics B, vol. 61, pp. 69-72, 1995. 
[7] J. Guézet and T. Kageyama, "Etude aérodynamique dans une machine à compression rapide," Revue Générale de Thermique, vol. 36, no. 1, pp. 17-25, 1997.

[8] D. Lee and S. Hochgreb, "Rapid Compression Machines: Heat transfer and suppression of corner vortex," Combustion and Flame, vol. 114, pp. 531-545, 1998.

[9] G. Mittal and C.J. Sung, "Aerodynamics inside a rapid compression machine," Combustion and Flame, vol. 145, pp. 160-180, 2006.

[10] C. Strozzi, J. Sotton, A. Mura and M. Bellenoue, "Characterization of a two dimensional temperature field within a rapid compression machine using a toluene planar laser induced fluorescence imaging technique," Measurement Science \& Technology, vol. 20, 2009.

[11] K.H. Tran, P. Guibert, C. Morin, J. Bonnety, S. Pounkin and G. Legros, "Temperature measurements in a rapid compression machine using anisole planar laser-induced fluorescence," Combustion and Flame, vol. 162, no. 10, pp. 3960-3970, 2015.

[12] M. Ben-Houidi, J. Sotton, A. Claverie, C. Strozzi and M. Bellenoue, "Application of high-speed PIV and toluene PLIF techniques to study aerodynamics and thermal stratification inside a flat piston Rapid Compression Machine," in 18th International Symposium on the Application of Laser and Imaging Techniques to Fluid Mechanics, Lisbon, Portugal, 2016.

[13] E. Monteiro, J. Sotton, M. Bellenoue, N. Afonso-Moreira and S. Malheiro, "Experimental study of syngas combustion at engine-like conditions in a rapid compression machine," Experimental Thermal and Fluid Science, vol. 35, pp. 1473-1479, 2011.

[14] P. Guibert, A. Keromnes and G. Legros, "An experimental investigation of turbulence effect on the combustion propagation in rapid compression machine," Flow Turbulence Combust, vol. 84, pp. 79-95, 2010 .

[15] C. Strozzi, A. Mura, J. Sotton and M. Bellenoue, "Experimental analysis of propagation regimes during the autoignition of a fully premixed methane-air mixture in the presence of temperature inhomogeneities," Combustion and Flame, vol. 159, no. 11, pp. 3323-3341, 2012.

[16] C. Strozzi, A. Delicourt, M. Bellenoue and J. Sotton, "High Speed PIV Analysis of the Combustion Regimes During Autoignition of Homogeneous Fuel - Air Mixtures in a RCM," in 26th ICDERS, Boston, MA, USA, 2017.

[17] J.F. Griffiths, J.P. MacNamara, C. Mohamed, B.J. Whitaker, J. Pan and C.G.W. Sheppard, "Temperature fields during the developpement of auto-ignition in a Rapid Compression Machine," Faraday Discuss., vol. 119, pp. 287-303, 2001.

[18] J.F Griffiths and B.J. Whitaker, "Thermokinetic interactions leading to knock during homogeneous charge compression ignition," Combustion and Flame, vol. 131, no. 4, pp. 386-399, 2002.

[19] J.F. Griffiths, J.P. MacNamara, C.G.W. Sheppard, D.A. Turton and B.J. Whitaker, "The relationship of knock during controlled autoignition to temperature inhomogeneities and fuel reactivity," Fuel, vol. 81, pp. 2219-2225, 2002.

[20] P. Desgroux, R. Minetti and L.R. Sochet, "Temperature distribution induced by pre-ignition reactions in a Rapid Compression Machine," Combustion Science and Technology, Vols. 113-114, pp. 193-203, 1996.

[21] A. K. Das, M. Uddi and C. J. Sung, "Two-line thermometry and H2O measurement for reactive mixtures in rapid compression machine near 7.6 m," Combustion and Flame, vol. 159, pp. 3493-3501, 2012.

[22] E. F. Nasir and A. Farooq, "Time-resolved temperature measurements in a rapid compression machine using quantum cascade laser absorption in the intrapulse mode," Proceedings of the Combustion Institute, vol. 36, pp. 4453-4460, 2017.

[23] C. Strozzi, J. Sotton, A. Mura and M. Bellenoue, "Experimental and numerical study of the influence of temperature heterogeneities on self-ignition process of methane-air mixtures in a Rapid Compression Machine," Combustion Science \& Technology, vol. 180, pp. 1827-1855, 2008.

[24] E. Monteiro, J. Sotton, M. Bellenoue, N. Afonso-Moreira and S. Malheiro, "Experimental study of syngas combustion at engine-like conditions in a rapid compression machine," Experimental Thermal and Fluid Science, vol. 35, pp. 1473-1479, 2011.

[25] A. Sciacchitano, F. Scarano and B. Wieneke, "Multi-frame pyramid correlation for time-resolved PIV," Exp Fluids, no. 53, pp. 1087-1105, 2012.

[26] L. M. Pickett, C. L. Genzale, G. Bruneaux, L-M. Malbec, L. Hermant, C. Christiansen and J. Schramm, "Comparison of diesel spray combustion in different high-temperature, high-pressure facilities," $S A E$, 2010-01-2106, 2010 
[27] M. Meijer, B. Somers, J. Johnson, J. Naber, S-Y. lee, L-M. Malbec, G. Bruneaux, L. Pickett, M. Bardi, R. Payri and T. Bazyn, "Engine Combustion Network (ECN): characterization and comparison of boundary conditions for different combustion vessels," Atomization and Sprays, vol. 22, no. 9, pp. 777 806, 2012.

[28] M. Tagawa, K. Kato and Y. Ohta, "Response compensation of fine-wire temperature sensors," Review of scientific Instruments, vol. 76, no. 9, 2005.

[29] P. Paranthoen, J.C. Lecordier, C. Petit and P. Gajan, "Survey \& recent developments of frequency response studies of cold wires \& fine wire thermocouples in turbulent heated flow," in Eighth Australasian Fluid Mechanics Conference, , University of Newcaste, N.S.W., 1983.

[30] M.V. Heitor and A. L. N. Moreira, "Thermocouples and sample probes for combustion studies," Prog. Energy Combust. Sci., vol. 19, pp. 259-278, 1993.

[31] G. Arwatz, C. Bahri, A. J. Smits and M. Hultmark, "Dynamic calibration and modeling of cold wire for temperature measurement," Meas. Sci. Technol., vol. 24, 2013.

[32] H. Arlaud and G. Lalizel, "Caracterisation spectrale de microthermocouples K de 7.6 micrometres de diametre pour une application aux mesures de fluctuations de temperature dans les ecoulements anisothermes turbulents," in Congres Francais de thermique, Toulouse, France, 31 Mai to 3 June, 2016.

[33] P. Paranthoen and J.C. Lecordier, "Mesures de temperature dans les ecoulements turbulents," Rev. Gen. Therm., vol. 35, pp. 283-308, 1996.

[34] W. M. Pitts, E. Braun, R. D. Peacock, H. E. Mitler, E. L. Johnsson, P. A. Reneke and L. G. Blevins, "Temperature Uncertainties for Bare-Bead and Aspirated Thermocouple Measurements in Fire Environments," Thermal Measurements: The Foundation of Fire Standards, ASTM STP 1427, L.A. Gritzo and N. J. Alvares, Eds., West Conshohocken, PA, 2002.

[35] F. R. Caldwell, Thermocouple materials, National Bureau of Standards Monograph No. 40, 1962.

[36] G. Mittal and M. Chomier, "Interpretation of experimental data from rapid compression machines without creviced pistons," Combustion and Flame, vol. 161, pp. 75-83, 2014.

[37] Zeldovich Ya.B., Combustion and Flame, vol. 39, pp. 211-214, 1980. 